\title{
3. Nationaler Grippeimpftag der Schweizer Hausärzte/-ärztinnen am 10. November 2006
}

Die hausärztlichen Organisationen (KHM, SGAM, SGIM, SGP und FMP) rufen für die dritte Durchführung des Nationalen Grippeimpftages am Freitag, den 10. November 2006, auf.

Nach wie vor ist die Grippeerkrankung in der Schweiz die Infektionskrankheit, die am meisten Komplikationen und die höchste Sterblichkeit verursacht. Das wirksamste Mittel, um diese Erkrankung zu verhindern und deren Komplikationen zu vermindern, ist die Grippeimpfung.

Ursula Laubscher ${ }^{a}$ und Ueli Grüninger ${ }^{b}$

a Koordinatorin Grippeimpftag KHM

b Geschäftsführer KHM

Einerseits die Gruppe mit erhöhtem Erkrankungs- und Komplikationsrisiko (d.h. Personen über 65 Jahre, chronisch Kranke, Heimbewohner/innen), andererseits jene der Überträger/innen, die diese besonders Erkrankungsgefährdeten leicht anstecken könnten (Medizinal-, Pflegeund Betreuungspersonal)

Korrespondenz:

Kollegium für Hausarztmedizin KHM Ursula Laubscher,

Koordinatorin Grippeimpftag

Dr. Ueli Grüninger,

Geschäftsführer

Landhausweg 26

CH-3007 Bern

Tel. 03137006 70/71/72

Fax 0313700679

ursula.laubscher@kollegium.ch

ueli.grueninger@hin.ch

www.kollegium.ch
Nur eine kontinuierliche und fortwährende Information über die Folgen und möglichen Komplikationen der Grippeerkrankung kann die Bevölkerung für die Grippeimpfung sensibilisieren und motivieren. Jährlich erkranken in der Schweiz im Durchschnitt 300000 Personen an der saisonalen Grippe, die sich normalerweise zwischen November und April bemerkbar macht. Zwischen 400 und 1000 Personen sterben an den Folgen dieser Erkrankung und zwischen 1000 bis 5000 Personen werden infolge der Erkrankung hospitalisiert. Besonders gefährdet sind die Personen über 65 Jahre und Personen mit bestehenden chronischen Krankheiten, weshalb vor allem diese Gruppe von Risikopersonen* erreicht werden soll. Mit der Grippeimpfung, deren Impfstoff jährlich den möglichen Virusvarianten angepasst wird, lässt sich das Risiko einer Erkrankung erheblich reduzieren. So zeigen vergleichende Untersuchungen zwischen nichtgeimpften und geimpften Risikopersonen, dass geimpfte Risikopersonen eine deutliche Abnahme der Komplikationen und der Nebenwirkungen einer Grippe aufweisen.

Unter den geimpften Risikopersonen sind Pneumonien um ein Drittel und bakterielle Pneumonien um 50\% weniger häufig. Ausserdem vermindert die Grippeimpfung die Sterblichkeit der geimpften Risikopersonen bei einer Grippe um über 50\%; und schliesslich sind die grippebedingten Hospitalisationen um 46\% reduziert [1].

Viele Hausärzte/-ärztinnen impfen ihre Risikopersonen bereits zu Beginn der Grippesaison. Die Impfung ist ein altbewährtes und etabliertes Instrumentarium für den Grippeschutz.

Wozu also noch ein Nationaler Grippeimpftag? Durch den Nationalen Grippeimpftag der Schweizer Hausärzte/-ärztinnen wird die Grippeimpfung zum sichtbaren Event, der die Bevölkerung sukzessive über die Grippeerkrankung informiert und aufklärt. Gleichzeitig wird der aktuellen, epidemiologischen Situation Rechnung getragen und die nötige Beachtung geschenkt. Mit diesem präventivmedizinischen Angebot der Grundversorger soll jenen Risikopersonen der Zugang zur Impfung ermöglicht und erleichtert werden, die sich nicht in kontinuierlicher medizinischer Betreuung befinden. Längerfristiges Ziel ist es, die Durchimpfungsrate bei den Risikopersonen zu verbessern.

\section{Erfahrungen aus dem Grippeimpftag 2005} Durch die Vogelgrippe und die Pandemieangst ist der Verlauf der saisonalen Impfsaison massgeblich kompliziert worden. Bereits vor dem Grippeimpftag war der Höhepunkt einer vermehrten und massiven Nachfrage der Impfung erreicht. Viele Personen ausserhalb der Risikogruppen - verunsichert durch die besondere Situation und die vielen Medienberichte - fühlten sich zur Impfung bewogen. Dadurch gab es in einigen Praxen eine Impfstoffknappheit, die die Ärztinnen und Ärzte spürbar miterlebten. Aus der Sentinella-Umfrage über die Impfstoffversorgung (Stichtag 9. Dezember 2005) geht hervor, dass $45 \%$ der Praxen zu wenig Impfstoff - zwar meist nur in geringem Ausmass - hatten, oder anders formuliert, fast jede zweite Praxis musste impfwillige Personen abweisen. Verständlich sind daher auch die Reaktionen, die von Verunsicherung bis zur Empörung reichten. Die Ärztinnen und Ärzte befanden sich in der ziemlich einmaligen, vermutlich erstmaligen Situation, dass ein medizinisches Gut nicht mehr zur Verfügung stand. Dies in einer Zeit, besser gesagt, 
in einem Kulturkreis, in dem sich die Gesundheit zum Konsumgut entwickelt hat und es als selbstverständlich angesehen wird, dass Krankheiten präventiv angegangen werden können. Kommt dazu, dass sich hier der Konsumanspruch auf das «first come - first served»-Prinzip in Frage gestellt sah. Der Umgang mit diesen beiden Konstellationen hat eine grosse Herausforderung bedeutet.

Aus epidemiologischer Sicht war der Mangel an Grippeimpfstoff hingegen nicht dramatisch: das zeigen die Resultate aus Erhebungen bei den Risikopersonen, wonach nur 3,1\% dieser Personengruppe oder knapp jeder 33. Risikopatient nicht geimpft werden konnte.

Hinter der festgestellten Impfstoffknappheit steckt tendenziell eher ein Verteilungsproblem. Es ist festzuhalten, dass die noch verfügbaren Impfstoffdosen am Grippeimpftag sehr unterschiedlich verteilt waren, dies in Abhängigkeit davon, ob es sich um ein Stadtgebiet oder um eine Agglomeration handelte, ob in der welschen, italienischsprechenden oder in der deutschen Schweiz. Mit anderen Worten: der vorhandene Impfstoff war nicht immer dort verfügbar, wo er nachgefragt wurde. Viele Ärzte haben deshalb mit erheblichem Aufwand Nachschub organisieren müssen.

Erfreulicherweise kann man aus der Sentinella-Auswertung aber trotzdem schliessen, dass erneut mehr Personen mit erhöhtem Risiko gegen Grippe geimpft worden sind. Die Zahl der zusätzlichen Grippeimpfungen am Grippeimpftag liegt bei 7,4 Personen pro Praxis im Mittel bzw. 2 Personen(Medianwert), im Vergleich dazu der Grippeimpftag 2004: mit 5,8 Personen pro Praxis im Mittel bzw. 3 Personen (Medianwert).

Diese Auswertungen verdeutlichen, dass die guten Ergebnisse nur dank des enormen Einsatzes der Ärztinnen und Ärzte zustande gekommen sind, und ihnen gebührt ein grosser Dank dafür!

Massnahmen für den Grippeimpftag 2006 Aus den Erfahrungen im 2005 sind die Impfstoffhersteller im Januar 2006 gebeten worden, ihre Impfstoffmenge entsprechend den Bedürfnissen anzupassen. Nach Mitteilung des BAG wird nun für 2006 11\% mehr Impfstoff als im Vorjahr produziert werden, das sind rund 180000 zusätzliche Dosen oder total 1,3 Mio. Dosen Impfstoff gegen die saisonale Grippe.

Aus den Erfahrungen des Grippeimpftages 2005 sind für den Grippeimpftag 2006 die folgenden Empfehlungen ausgearbeitet worden:

- Die Etappierung der Grippeimpfung: bis und mit Grippeimpftag 10. November 2006 sollen prioritär die Risikopatienten geimpft werden, um bei allfälligen Engpässen bei der Lieferung des Grippeimpfstoffes wenigstens diese bereits geimpft zu haben.

- Erst nach dem Grippeimpftag soll die Impfung den Personen ausserhalb der Risikogruppe verabreicht werden.

- Die begrenzten Impfstoffvorräte sollen zuerst den beiden Risikogruppen* zur Verfügung stehen.

Die Ankündigung des 3. Nationalen Grippeimpftages ist bereits Ende Januar 2006 an alle hausärztlichen Organisationen und an die Impfstofflieferanten kommuniziert worden. Diese frühzeitige Information über den Nationalen Grippeimpftag berücksichtigt die vielen Rückmeldungen und Anregungen der Grundversorger.

\section{Aktueller Stand bezüglich Verfügbarkeit der Impfstoffmenge}

Allerdings haben die Herstellerfirmen schon vor einiger Zeit gewisse Lieferverzögerungen wegen Produktionsengpässen bei einer Impfstoffkomponente angekündigt.

Aus den Meldungen der Impfstoffproduzenten und der öffentlichen Stellen ergibt sich heute folgendes Bild:

- die Auslieferung des Impfstoffes wird durch die Komplexität des Produktionsprozesses etwas verzögert;

- für die Risikopersonen wird der Impfstoff so rechtzeitig zur Verfügung stehen, dass alle Risikopersonen bis zum Grippeimpftag 2006 geimpft werden können.

\section{Teilnahme am Grippeimpftag 2006}

Mehr als die Hälfte aller Praxen hat im 2005 teilgenommen, also etwa gleichviel wie 2004 - und dies trotz erschwerter Umstände mit Engpässen bei der Impfstoffverteilung. Auch am dritten Grippeimpftag 2006 will die überwiegende Zahl der im 2005 teilnehmenden Praxen wiederum teilnehmen.

Diese Zahlen belegen die Einsatzbereitschaft der Grundversorger - dies wohlbemerkt unter besonderen Umständen - und spiegeln auch die Überzeugung der Ärzte und Ärztinnen wider, sich für präventivmedizinische Massnahmen einzusetzen. Es ist ein wichtiger Beitrag an die Gesundheit der Bevölkerung.

Literatur

1 Hatz C. Über Epidemien ohne Grenzen - Gefahr oder Medienhype? Hauptvortrag an der Fortbildungstagung des KHM, 1. Juni 2006 in Luzern. 